\title{
Development of Myocardial Sympathetic Innervation in the Fetal Lamb
}

\author{
Edward A. Lebowitz, James S. Novick, and Abraham M. Rudolph ${ }^{[20]}$ \\ Cardiovascular Research Institute and Department of Pediatrics, University of California, San Francisco, California, USA
}

\begin{abstract}
Extract
The hearts of ten fetal lambs with gestational ages ranging from 75 days to term (150 days) and of two neonatal lambs were studied by the Falck-Owman monoamine fluorescence technique to determine the pattern of development of the sympathetic nervous system. At 75-85 days of gestation, no sympathetic fibers are present in the heart, but brightly fluorescent dopamine-containing cells are diffusely scattered throughout the myocardium. Between 75-85 and 100-110 days, large nerve trunks begin to grow in along the coronary arteries and small branches are first distributed to the coronary arteries and later to the myocardium. There is continued growth of sympathetic fibres and, by term, an extensive network exists that supplies a large number of myocardial cells. Some growth occurs after birth, and continued innervation probably persists for several weeks. These findings are helpful in explaining the physiological observations of increasing autonomic activity in the circulation of the fetal lamb, and they suggest that growth of myocardial sympathetic nerves is one of the final steps in development of cardiovascular control.
\end{abstract}

\section{Speculation}

The innervation of the myocardium by sympathetic fibers is incomplete before birth in the lamb. If the same pattern of development is present in the human fetus and infant, it is very likely that the circulation of the premature infant lacks the ability to respond to stress through sympathetic stimulation.

\section{Introduction}

Neural control of myocardial contractility and heart rate requires the presence of myocardial sympathetic innervation. In different species this is established at different ages. For example, rabbit [10] and rat [14] hearts are almost devoid of sympathetic fibers before birth, whereas guinea pig [12] hearts contain them before the primitive mesenchymal tissue has fully differentiated to definitive myocardium. Unfortunately, neither physiologic nor pharmacologic studies are available for these fetuses. For the fetal lamb, however, such studies are available and these indicate that neural cardiovascular control develops largely during late gestation $[5,16,17]$. Furthermore, they suggest that progressive neural development rather than increasing end-organ responsiveness is responsible for this. To determine the role of myocardial sympathetic development in this process, we studied the hearts of fetal and neonatal lambs by the Falck-Owman monoamine fluorescence technique $[4,8]$.

\section{Methods}

The hearts of 10 fetal and 2 neonatal lambs were studied. The gestational ages of the fetuses, as estimated 

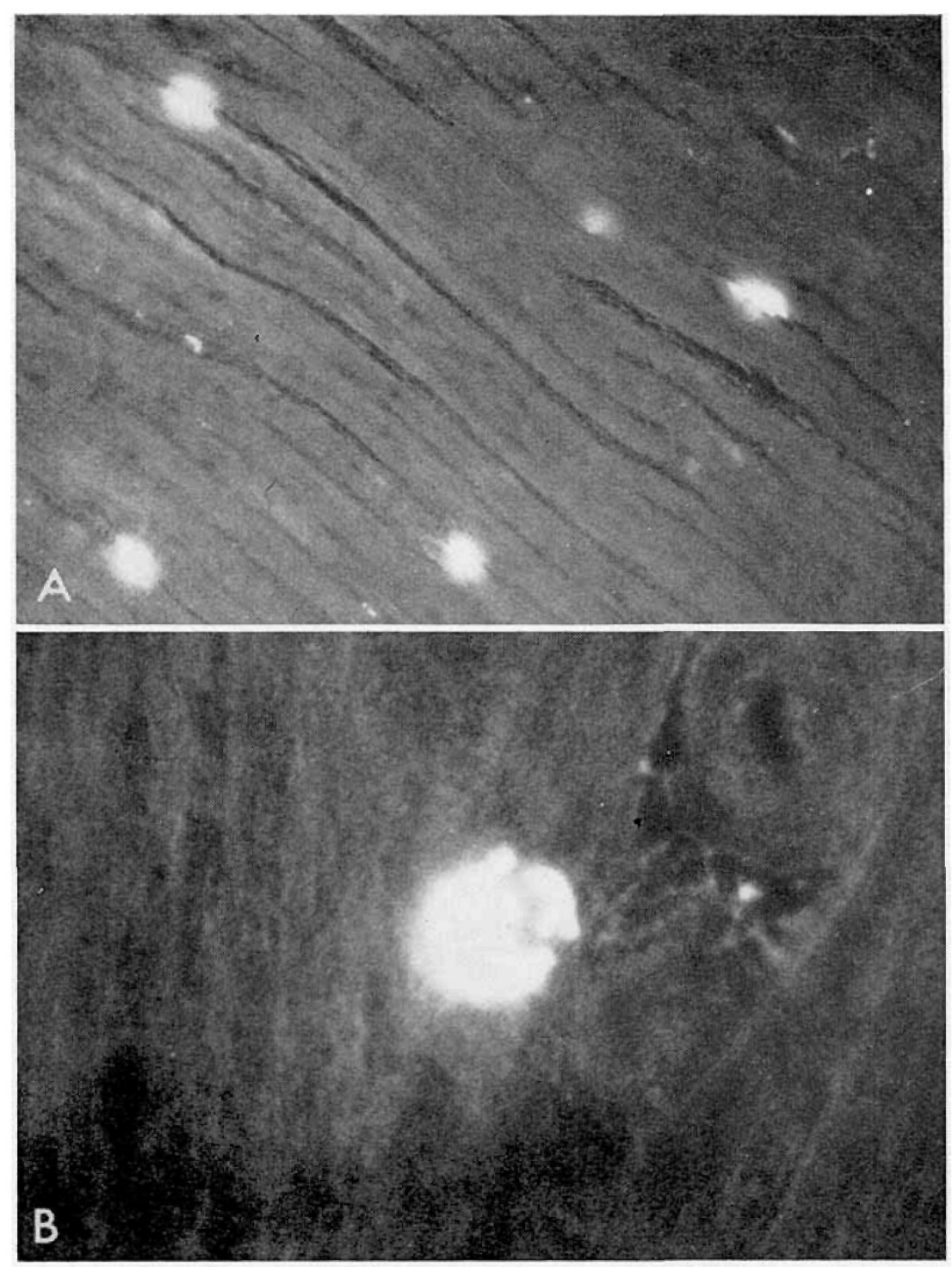

Fig. 1. A: Myocardium of 75-day gestation lamb. Note that although several dopamine-containing cells are visible in the 75-day heart, no sympathetic fibers are present. B: Dopamine-containing cell. $\times 400$.

from fetal body weight [1], ranged from midgestation (75 days) to term (150 days). Specific ages were 75,80 , $85,100,108,110,124,130,148$, and 150 days. The fetuses were obtained by hysterotomy in ewes after low spinal anesthesia with $20 \mathrm{mg}$ tetracaine (Pontocaine). The two neonatal lambs were anesthetized with 300 $\mathrm{mg}$ pentobarbital administered intravenously. The hearts were removed immediately and several tissue samples were excised. These were immersed in liquid freon pooled with liquid nitrogen and then freeze dried at $-35^{\circ}$ for 5 days. Subsequently, they were exposed to formaldehyde vapor at $80^{\circ}$ for $1 \mathrm{hr}$. The formaldehyde was generated from paraformaldehyde equilibrated in air with about $70 \%$ relative humidity.
The tissue samples were then vacuum embedded in paraffin at $60^{\circ}$ for $15 \mathrm{~min}$. Serial sections 5-, 10-, and $15-\mu$ thick were studied for fluorescent structures containing primary catecholamines. To distinguish fluorescence caused by norepinephrine from that caused by dopamine, several paraffinized sections were exposed to $\mathrm{HCl}$ vapor at room temperature for $\mathrm{l}-1.5 \mathrm{hr}$. This causes the fluorescence due to norepinephrine to disappear on exposure to standard excitation conditions while fluorescence due to dopamine remains [2].

\section{Results}

Structures containing catecholamine were noted in the 75- and 85-day gestation lamb hearts. These were in- 


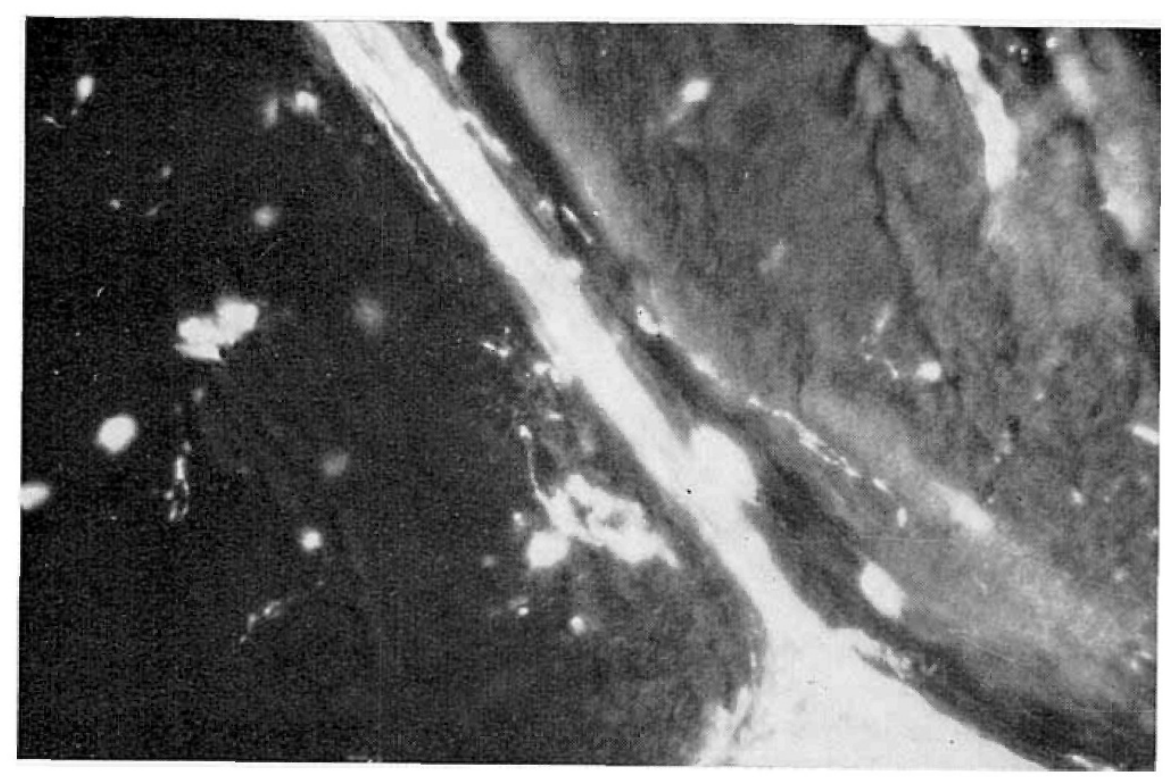

Fig. 2. Nerve trunk giving rise to terminal sympathetic fibers. $\times 160$. See text for details.

tensely fluorescent cells containing dopamine (Fig. $1 A$ ). Although most prevalent subepicardially, these cells occurred throughout the atria and ventricles. They were seen not only in myocardial muscular tissue but also in myocardial adipose tissue, fibrous tissue, and in close association with coronary vessels. The cells were usually single but occasional clusters were noted. They were ellipsoidal or round with a diameter of 10-15 $\mu$. Some of the cells possessed short branches which appeared as beaded filaments. The dopamine in these cells was present only within intracytoplasmic granules (Fig. 1B). Neither the appearance nor distribution of these cells changed from midgestation to birth.

In the hearts of fetuses of 100- to 110-day gestation, thick nerve trunks containing norepinephrine were seen coursing along the epicardium and through the myocardium, usually in the adventitia of coronary vessels. These contained fluorescent varicosities associated with terminal sympathetic fibers and these varicosities were responsible for the fluorescence of the nerve trunks. With advancing gestation, these trunks became more intensely fluorescent. Figure 2 demonstrates such a trunk giving rise to a smaller trunk in the heart of an 18-hr-old neonatal lamb. Peeling away from the smaller trunk are terminal sympathetic fibers with fluorescent varicosities. When they first appeared, these trunks were associated with a scanty network of fluorescent fibers containing norepinephrine which surrounded the larger coronary vessels and myocardial muscle bundles. This network grew denser throughout gestation and, by term, sympathetic fibers had formed a matrix which was associated with every myocardial muscle cell, the smallest arterioles and venules, and parasympathetic ganglion cells. Innervation of coronary vessels was more developed than that of myocardial muscle fibers throughout gestation. Figures 3, 4, and 5 illustrate these findings. The time course of development of sympathetic innervation in the coronary vessels and myocardium is graphically depicted in Figure 6.

\section{Discussion}

Falck et al. [7] has previously demonstrated the presence of granulated, fluorescent mast cells in several tissues of cows and sheep. By measurement of concentrations of dopamine and norepinephrine in tissue, he showed that the presence of these cells correlated with dopamine and not with norepinephrine. He inferred from this that dopamine rather than norepinephrine was responsible for the fluorescence of these cells. We have confirmed this in the fetal ovine heart with the selective fluorescence method. The function of these cells is as yet undefined. That fact that dopamine is found stored in them does not necessarily indicate that they secrete this specific catecholamine. It is possible that they secrete dopamine, but it is also possible that the catecholamine they secrete is norepinephrine, since a single enzyme, dopamine- $\beta$-oxidase, can convert dopamine to norepinephrine [11]. It would be interesting if they secreted dopamine as this does have a positive inotropic and chronotropic effect on the dog heart [15]. The occurrence of both norepinephrine and do- 


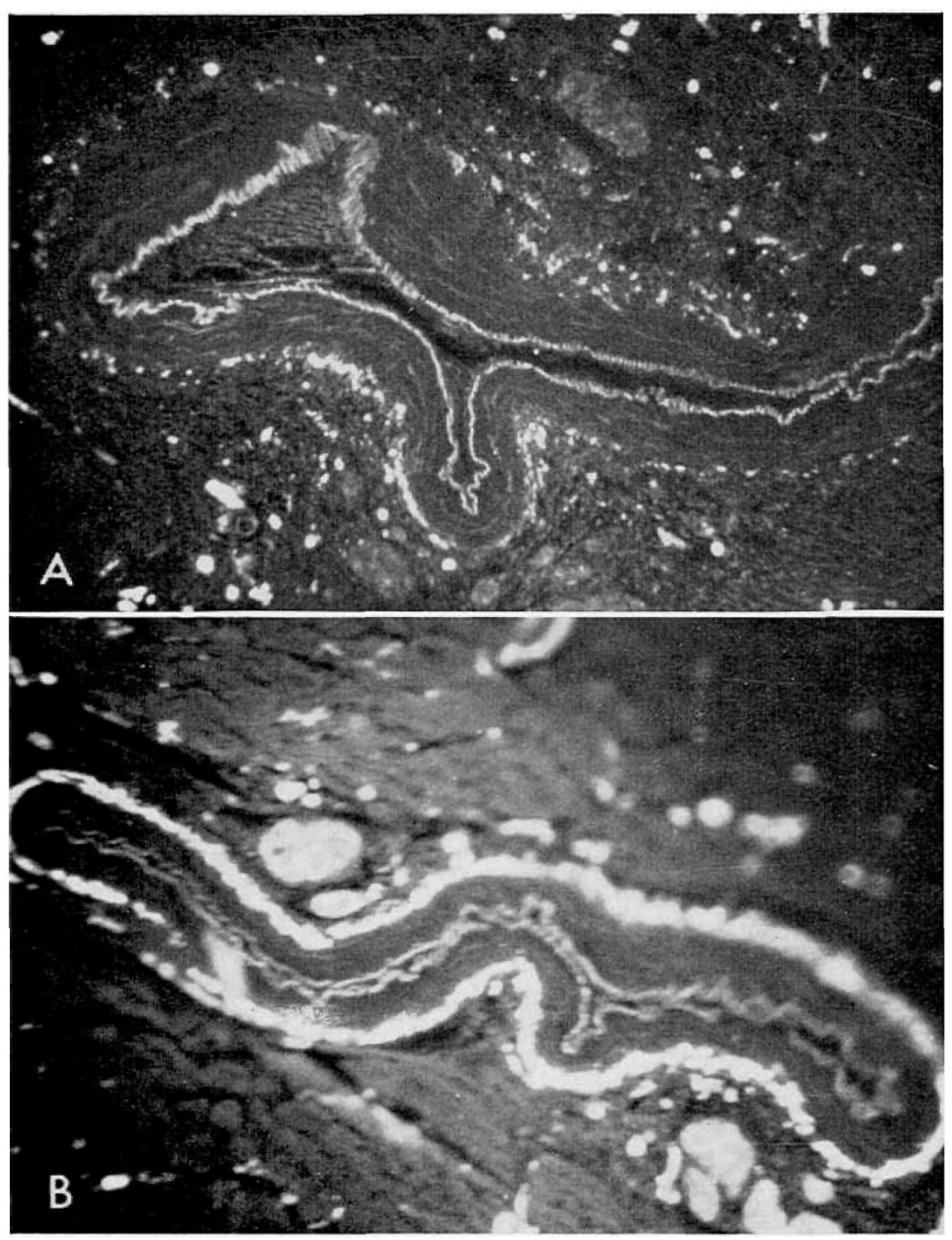

Fig. 3. Development of coronary vascular innervation and increasing fluorescence of nerve trunks. $\times 100 . A: 100-$ day fetus. $B:$ Newborn Note the increase in nerve trunk fluorescence and thickness of layer of sympathetic fibers surrounding the medial layer. Note the presence of dopamine-containing cells in both sections. Note the autofluorescent elastic fibers in the intimal and medial layers.

pamine within these cells could not be assessed as present fluorescence methods do not allow for detection of both catecholamines simultaneously. It is possible that the presence of dopamine- $\beta$-oxidase within these cells could permit the local secretion of norepinephrine in the midgestation fetal lamb heart before any sympathetic fibers are detectable. The mechanism by which catecholamine could be released, in the absence of nerve supply, is not evident. In fact, it has not been shown that these cells possess any secretory function. In this regard, it is of considerable interest that $\beta$-receptor blockade decreases heart rate in unstressed fetal lambs of less than 100-day gestation, a time when little sympathetic innervation is present [17]. This would suggest that either norepinephrine or another catecholamine is secreted from a nonneural site in amounts sufficient to maintain continuous stimulation of the heart. The data of Comline et al. [3] indicate that the resting secretory rate of the adrenal medulla of the fetal lamb is low and that substantial secretion is enacted only when descending aortic oxygen tension falls below normal. This suggests that the adrenal medulla 


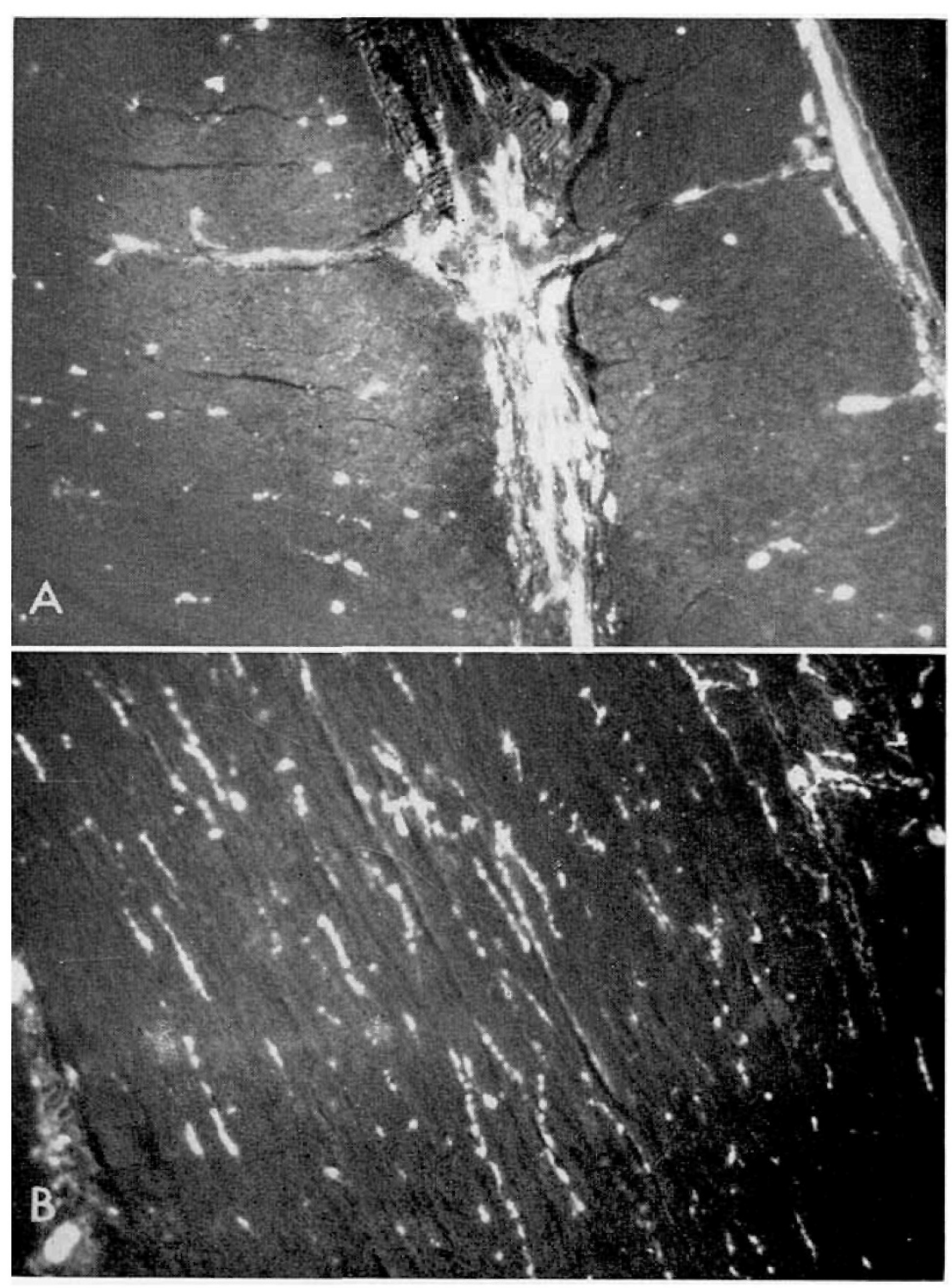

Fig. 4. Development of myocardial muscular innervation. $\times 100 . A: 100$-day fetus. $B:$ Newborn. Note the fluorescent nerve trunk in the epicardium of the 100-day heart. Note also that the network of fibers surrounding the coronary vessel is already quite dense while the innervation of the myocardial muscle cells themselves is still sparse. Note the increasing density of myocardial muscular innervation from 100 days of gestation to birth.

is not the site of catecholamine release. However, this finding may be explained if the dopamine cells do have a secretory function and if their activity before cardiac innervation is substantial.

The pattern of sympathetic nerve fiber growth into the fetal lamb heart is similar to that reported for rabbits by Friedman et al. [10], and this pattern has been confirmed in guinea pig and rat in our laboratory. That is, sympathetic fibers appear to grow into the heart through large nerve trunks rather than to form in situ from structures within the embryonic or fetal myocardium. Figure 2 illustrates terminal sympathetic fibers which were growing into the heart of one neonatal lamb. The time course of growth, however, is different in the various species. Whereas, in rabbit and rat, the major development is postnatal, in lamb, myocardial sympathetic innervation is already well developed at birth. Growth of sympathetic fibers into the lamb heart probably continues for weeks and possibly even months after birth. Consistent with our observa- 


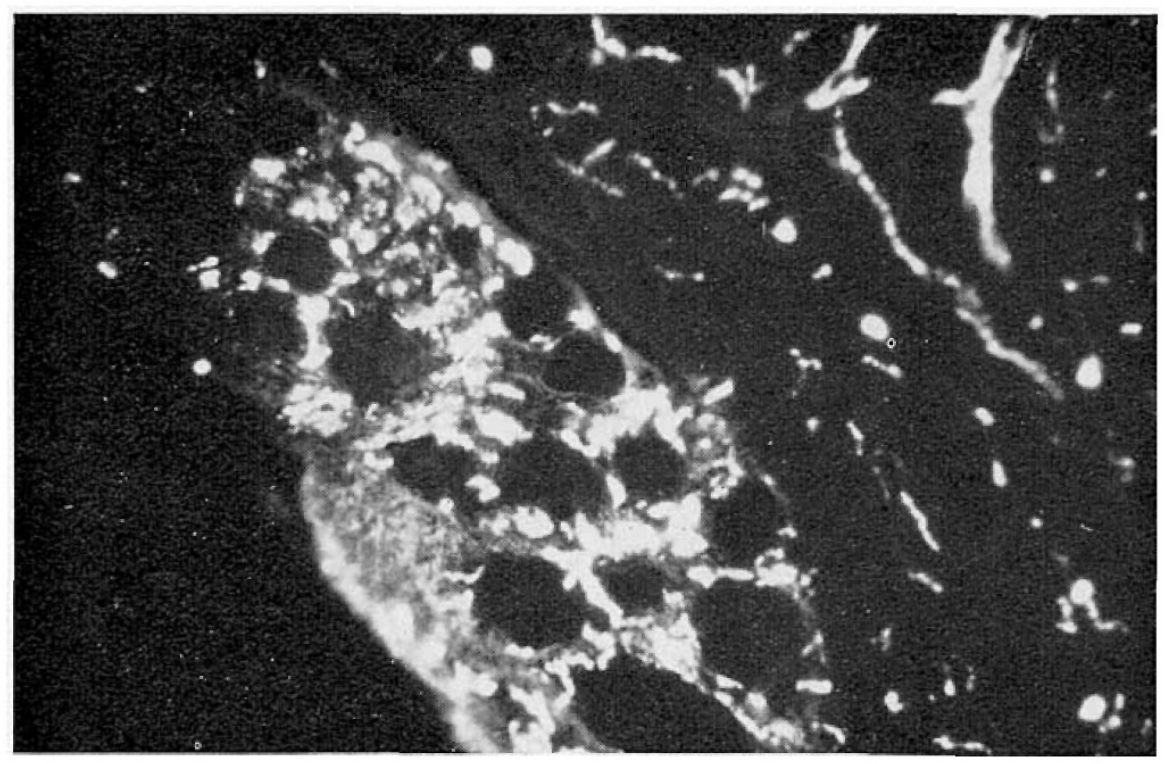

Fig. 5. Sympathetic innervation of parasympathetic ganglion cells.

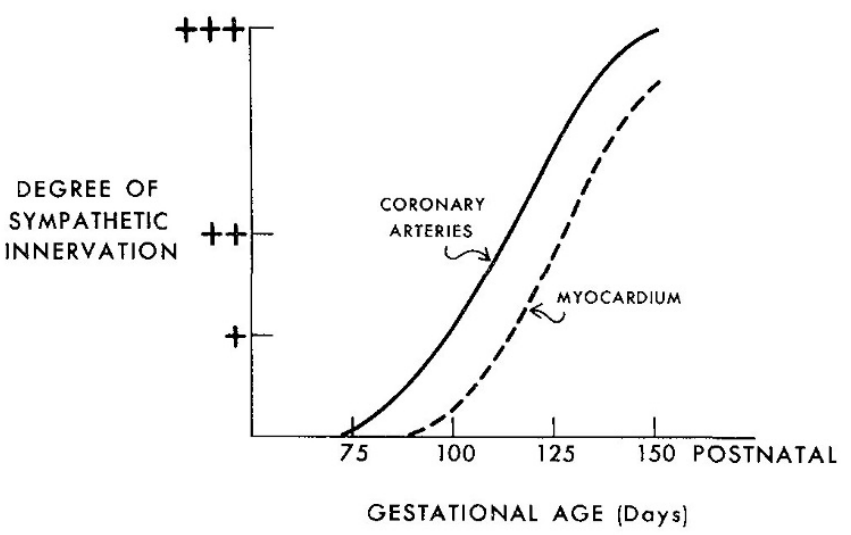

Fig. 6. Graphic portrayal of development of myocardial sympathetic innervation.

tions, Vogel et al. [18] found the density of sympathetic fibers in neonatal calf hearts essentially the same as that in adult cow hearts. As noted previously, coronary vascular innervation exceeded myocardial muscular innervation throughout most of gestation. Nerve trunks through which sympathetic fibers grow into the heart lie close to coronary vessels, usually in their adventitial layers. Consequently, the distance a sympathetic fiber must travel to innervate the vessel is less than the distance it must travel to innervate surrounding myocardial muscle cells. Our data conflict with those of Friedman et al. [9] who could demonstrate only sparse sympathetic innervation of the fetal lamb heart at term when they used the same histochemical technique as we did.
Our findings help to explain the observations of Downing et al. [6], who found that the positive inotropic and chronotropic response to supramaximal cardiac sympathetic nerve stimulation in newborn lambs did not differ from that observed in adults of many species. They also found no differences in responses to tyramine infusion of the hearts of newborn and of 2-month-old lambs.

Although study of the development of myocardial parasympathetic innervation and sympathetic-parasympathetic interconnections was not our object, we recognize their importance to the development of neural control of the heart [13]. In this regard, we demonstrated the presence of substantial sympathetic innervation of parasympathetic ganglion cells in the hearts of newborn lambs (Fig. 5). This suggests that autonomic innervation of the lamb heart at birth is qualitatively as well as quantitatively well developed.

Autonomic regulation of the cardiovascular system can be present only when receptors, afferent nerve pathways, central and peripheral ganglionic syna. $\mathrm{i}_{\mathrm{i}} \mathrm{ses}$, and efferent nerves have developed. Furthermore, both carrliac cells and vascular smooth muscle cells must respond to nervous stimulation. Dawes [5] has commented on the progressive increase in arterial blood pressure in the fetal lamb, noting a more rapid rise near term, and has suggested that this is due to the development of autonomic cardiovascular control. Studies of the effects of specific $\alpha$ - and $\beta$-receptor blocking drugs in unstressed lambs in utero also show evidence of a progressive increase in resting autonomic 
regulation after 100 days of gestation [17]. Furthermore, there is an increase in baroreceptor reflex sensitivity and response in the fetal lamb during the latter half of gestation [16]. The histologic studies which we have performed show that this progressive development of autonomic control of the cardiovascular system coincides with the development of myocardial sympathetic innervation. Although it is possible that there is simultaneous development of other aspects of the autonomic nervous system, it would appear that growth of myocardial sympathetic nerves is one of the final steps in the development of autonomic cardiovascular control in the fetal lamb.

\section{Summary}

The developmental pattern of the sympathetic nerve supply to the heart was studied histologically in 10 fetal lambs of 75-day (0.5) to 150-day (term) gestational age, using the monoamine fluorescence technique. There were scattered dopamine-containing cells in the myocardium of the 75- to 85-day fetuses, but no evidence of any sympathetic nerves. Large sympathetic fibers grow into the heart along the coronary arteries between 90 and 100 days of gestation. Branches are distributed first to the coronary arteries and then to the heart muscle. Innervation of the myocardium continues to develop throughout gestation and is well developed, though still not complete at birth. On the basis of these observations, it would appear that the ability of the heart in the fetal lamb to respond to stress through sympathetic stimulation is limited.

\section{References and Notes}

I. Barcrofr, J.: Researches on Prenatal Life. (Thomas, Springfield, Ill., 1964).

2. Bjorklund, A., Ehinger, B., And Falck, B.: A method for differentiating dopamine from noradrenaline in tissue sections by microspectrofluorometry. J. Histochem. Cytochem., 16: 263 (1968).

3. Comline, R. S., Silver, I. A., And Silver, M.: Factors responsible for the stimulation of the adrenal medulla during asphyxia in the foetal lamb. J. Physiol., 178: 211 (1965).

4. Corrodi, H., and Jonsson, G.: The formaldehyde fluorescence method for the histochemical demonstration of biogenic monoamines. J. Histochem. Cytochem., 15: 65 (1967).
5. Dawes, G. S.: Foetal and Neonatal Physiology, p. 91. (Year Book Medical Publishers, Inc., Chicago, 1968).

6. Downing, S. E., Talner, N. S., Campbell, A. G. M., HalloRAN, K. H., AND WAX, H. B.: Influence of sympathetic nerve stimulation on ventricular function in the newborn lamb. Circ. Res., 25: 417 (1969).

7. Falck, B., Nystedr, T., Rosengren, E., and Strenflo, J.: Dopamine and mast cells in ruminants. Acta Pharmacol. Toxicol., 21: 51 (1964).

8. FALCK, B., AND OWMAN, C.: A detailed methodological description of the fluorescence method for the cellular demonstration of biogenic monoamines. Acta Univ. Lund., 2: 7 (1965).

9. Frifdman, W. F., Pool, P. E., Jacobowitz, D., Levitu, M., Sonnenblick, E. H., and Braunwald, E.: Sympathetic innervation of the fetal heart: pharmacological, biochemical, and histochemical comparisons of fetal, neonatal, and adult myocardium (Abstract). Circulation, 36 (suppl. I1): 114 (1967).

10. Friedman, W. F., Pool, P. E., Jacobowitz, D., Seagren, S. C., AND Braunwald, E.: Sympathetic innervation of the developing rabbit heart. Circ. Res., 23: 25 (1968).

11. Goodman, L. S., and Gilman, A.: The Pharmacological Basis of Therapeutics, p. 422. (Macmillan, New York, 1965).

12. HoAr, R. M., AND Hall, J. L.: The early pattern of cardiac innervation in the fetal guinia pig. Amer. J. Anat., 128: 499 (1970).

13. LevY, M. N.: Brief reviews: sympathetic-parasympathetic interactions in the heart. Circ. Res., 29: 437 (1971).

14. Lipp, J. A. M.: The devclopment of the sympathetic nervous system in the heart of the rat and guinea pig (Unpublished observations).

15. McDonald, R. H., JR., And GoldberG, L. I.: Analysis of the cardiovascular effects of dopamine in the dog. J. Pharmacol. Exp. Ther., 140: 60 (1963).

16. Shinebourne, E., Vapaavuori, E., Williams, R., Heymann, M., AND Rudolph, A.: Assessment of systemic and pulmonary baroreceptor function in intact unanesthetized fetal and newborn lambs. Pediat. Res., 5: 424 (1971).

17. Vapaavuori, E. K., Shinebourne, E. A., Williams, R. L., HexMANN, M. A., AND Rudolph, A. M.: Cardiovascular responses to autonomic blockade in intact fetal and newborn lambs. Pediat. Res., 5: 425 (1971).

18. Vogel, J. H. K., Jacobowitz, D., and Chidsey, C. A.: Distribution of norepinephrine in the failing bovine heart: correlation of chemical analysis and fluorescence microscopy. Circ. Res., 24: 71 (1969).

19. Supported by Grant no. HL06285 from the National Heart and Lung Institute, National Institutes of Health.

20. Requests for reprints should be addressed to: ABrafram M. Rudolph, M. D., Room 1403 HSE, University of California at San Francisco, San Francisco, Calif. 94122 (USA).

21. Accepted for publication August 15, 1972. 